\section{MEDICAL RESEARCH COUNCIL UNIT FOR APPLIED PSYCHOLOGY}

\author{
By DR. K. J. W. CRAIK \\ Director of the Unit
}

$\mathrm{T}$ HE Medical Research Council Unit for Applied Psychology consists at present of eleven research workers who had previously been carrying out investigations in the Psychological Laboratory, Cambridge, principally on problems arising from the War, under the direction of Prof. F. C. Bartlett, who continues to have general supervision of the Unit. Of these, six are graduates in psychology, four in medicine and one in physiology. Until the end of the War it is likely that work of the type now in hand will continue. This has necessarily involved problems largely of an $a d$ hoc character, undertaken in response to requests from Service departments, but certain common principles have emerged, which may assist in guiding a fruitful approach to future and peace-time problems. These principles are that of suiting the job to the man, of suiting the man to the job, and of improving the man's performance.

The first involves mainly the design of instruments, machinery, lay-out and illumination of maps, panels, etc. These problems may be broadly divided into those of display and control. The first term is that used by Service scientific departments to cover the methods by which information is laid visually before any operator, whether on a map, a graph, a cathoderay tube or an instrument panel. Often the best type of display is a compromise, for example, between an instrument-panel so complex that its interpretation is slow or so simple that it gives insufficient information, or, in the case of a night-fighter aircraft panel, so brightly lit that it dazzles the pilot or so dim that it cannot be read. Psychological experiments, employing laboratory simulations of the real conditions, often enable the optimum type of display to be decided upon. Even where an optimum does not exist, a graph of the relation between, say, the distance of a plotter from a map and his accuracy in reading grid references will show a very steep rise in orror beyond a certain point, and thus indicate a definite practical limit to the viewing distance, as in certain work by Dr. H. N. Mackworth. There should be a great deal of scope for such work in peace-time industrial design, especially in ensuring easier operation and preventing accidents, for example, in instrument panels, indicators, information charts and graphs. General principles have emerged which narrow the field for ad hoc experiments. Exact methods of scoring efficiency in war-tasks, such as watch-keeping, which are of a boring but responsible nature, have shown ways of determining optimal spells of work. The effect of discomfort, fatigue and noise on such tasks is also being studied. These techniques for the measurement of human abilities may eventually provide useful ways of assessing the progress of patients recovering from physical or mental illness, and perhaps may also test innovations introduced by researches in preventive medicine.

On the motor side, the positions, forces and gearratios of handles and levers on guns and machinetools are usually compromises between the factors of speed and precision of operation, of simplicity and mechanical perfection or of psychological and physiological suitability. Here, again, particular cases are being dealt with by laboratory simulations with exact scoring of performance, and interesting principles of muscular action and sensory - motor co-ordination are emerging. These studies verge on physiology and preventive medicine.

Similar problems arise in industrial design (such as the handwheels on a machine-tool, the stage at which servo-motor or remote control becomes necessary, and the most suitable form for such control), while general principles of use to designers in less important cases could be formulated.

Any human act can be regarded as the result of a sensory - mental - motor chain of events, and hitherto those in the Unit who have worked on the above problems have concentrated rather on the sensory and motor ends of this chain, partly because of their individual aptitudes but partly, perhaps, because these are the most fruitful sites for instrumental modification. There is probably, however, an interesting future field in the analysis of the factors that make a task intellectually difficult and have led industrialists to division of labour, with its advantages of increased output and its disadvantages of boredom and discontent.

This approach-suiting the job to the manshould, we feel, be explored to the full, since it puts the industrial jobs necessary for improved standards of living within the power of the majority, whereas psychological selection alone, especially when the job has been made unnecessarily difficult, may result in a high rate of rejection and unemployment. There is, however, need for allocation of the available workers so that they are given tasks for which they are suited, and some selection where a task is unavoidably difficult.

This second approach - suiting the man to the jobis principally being tackled by a team under Dr. A. W. Heim. They are members of the Unit, but are working on behalf of the Industrial Health Research Board of the Medical Research Council. They have devised a battery of tests and standardized it on a large number of entrants to factories and Government training centres and some university undergraduates, and are obtaining follow-ups on the industrial subjects. This battery contains a paper test (AH4) consisting of a verbal and arithmetical, and a visual part based on relations of identity and opposition, analogy, completing series, and following instructions; a mechanical ability test, an inspection test consisting of metal blocks containing small defects, performance being scored on an accuracy index, speed also being recorded; some other performance tests resembling factory gauging and assembly tasks, and the National Institute of Industrial Psychology Form Relations test. The emphasis of such work must necessarily be on individual differences in ability, rather than on the features of a task which make it difficult to everyone. Research is being carried out on consistency and validity of test results and of assessments, and on the relation between these two criteria. The extent to which the value of a test depends on its degree of analogousness and the distinction between differences of grade and type of ability are also being studied.

Certain members of the Unit are investigating night vision and other tests from the point of view of selection and of diagnosing vitamin-deficiency and disease. It is hoped that there will also be some time for fundamental work on the special senses. Mr. E. Farmer has begun a study of the capacities of blinded Service personnel with the view of their obtaining suitable employment. Others are using methods which link almost equally with both the two 
main approaches discussed above. For example, Miss M. L. Vernon is working on visual form perception and memory with the view both of lay-out of visual tasks and the allocation of personnel, and Dr. D. R. Davis is investigating the sensory motor co-ordination and responses of temperamentally different types of people in controlling machinery.

The third main approach is to improve the performance of the man, either by nutritional means or by mental and physical training. This, again, involves exactly controlled experimental tests of sensory and motor efficiency and scoring of performance. Various synthetic training devices have also been produced for Service use. Similar devices may well have a peace-time application, for example, to motor-car driving or machine-tool operation, where the novice is apt to injure either himself or the machine and to gain little insight into what he is doing wrong. Synthetic training equipment with exact scoring devices can assist here; but psychological experiments should always be undertaken to see whether any given trainer is in fact saving training time and improving performance, and whether it would do so equally well if it were simplified, or very much better if it were slightly complicated.

All these lines of approach involve much statistical work-for example, to establish the significance of the optimum values found for some feature of instrument design and to reveal the consistency or inconsistency of an allocation test, and its validity as judged by follow-up evidence. Though most of the members do the simpler statistical treatment of their own results, Mr. E. G. Chambers and Mr. J. W. Whitfield help in applying more complicated methods and in the development of new ones. Mr. Whitfield is also instituting a new type of recording system in a group of coal mines and two factories with the view of tracing causes of absenteeism, sickness and accidents. Such investigations indicate where there is a definite field for experimental research into improved equipment or for re-allocation or re-training of accident-prone workers.

It is hoped that there will be close contact with other bodies undertaking similar work-for example, the National Institute of Industrial Psychologyand with personnel managers, safety-officers and medical officers in factories. It must be emphasized, however, that the Unit is primarily a research body and has not the personnel or time for investigations of purely specific and local interest. Thus Dr. Heim has introduced, in several factories for which she has worked, a scheme by which members of her team inspect the problem and decide what existing test would seem appropriate and develop new ones if necessary ; the firm then provides a suitable person who is trained for a fortnight or so at Cambridge, and returns to the firm to give the tests and forward the results at intervals to Cambridge. Similarly, it will be impossible to take on a large number of particular problems in display or control design; but wherever a problem of wide interest arises, or one involving test-methods which could be applied as routine elsewhere when once they have been developed, the Unit is very anxious to be of assistance to any firms who raise them; and it is hoped that similar work may continue for the Services in regard to their more fundamental problems. The essential thing is that the scientific abilities of the members for basic research which ought, sooner or later, to have its effect on particular problems, should not be swamped by work of transitory and local value.

\section{MODELS OF THE UNIVERSE AND COSMOLOGICAL TIME-SCALES}

\author{
By DR, G. C. McVITTIE \\ King's College, London
}

$\mathrm{T}$ HE two time-scales, one 'dynamical' and the other 'cosmological', introduced by Prof. E. A. Milne into his theory of the structure of the universe, have certain curious biological consequences pointed out in Nature by Prof. J. B. S. Haldane'. Another time-scale has just been put forward by Sir Arthur Eddington ${ }^{2}$ on the basis of his unification of general relativity and quantum theory. It is therefore an opportune moment to attempt a general survey of cosmological investigations and, in particular, to direct attention to the very special assumptions on which the results of such inquiries depend. I do not believe that it is possible to give an accurate account of cosmological theory without expressing oneself to some extent in mathematical terms, and I trust that the reader will forgive this necessary evil-if evil it is.

\section{Models of the Universe}

Cosmological theories, whether connected with general, or with Milne's kinematical, relativity, start from the conception of an ideal universe which we shall call a 'model of the universe'. Essentially this may be regarded as a geometrical model in which the observed aggregate of spiral nebulæ is idealized into a set of mathematical points tracing out certain curves called 'geodesics' in a 4-dimensional spacetime. This space-time possesses a 'metric' of the general form

$d s^{2}=d t^{2}-e^{g(t)}\left\{\frac{d r^{2}}{1-k r^{2} / R_{0}{ }^{2}}+r^{2} d \theta^{2}+r^{2} \sin ^{2} \theta d \varphi^{2}\right\}$

Here $r, \theta, \varphi$ are space-co-ordinates, $t$ is a time-coordinate*, and $k, \boldsymbol{R}_{0}$ are constants which, together with the undetermined function $g(t)$, we leave for later discussion, only remarking here that ( 1 ) defines not one, but a whole class, of space-time models.

To arrive at this formula we need no theory of gravitation or of dynamics: we require only the following assumptions ${ }^{3}$. First, the aggregate of spiral nebulæ must form a homogeneous aggregate, which means that the nebulæ must be uniformly distributed. in space at each 'instant' $t$; secondly, each nebula must trace out a geodesic of space-time; and thirdly, these geodesics must form a 'coherent' set. The last assumption means that the aggregate of moving nebulæ is imagined as having had a continuous past history and not as having been formed by the fusion of two or more independent streams of nebulæ. In short, the metric (1) is derived from kinematic considerations together with the hypothesis of uniformity of distribution in space of the nebulæ.

Apart, howevier, from differences in their theories of dynamics and gravitation, general and kinematical relativity differ at this preliminary stage also. One important difference is that general relativity presupposes only that a model of the universe must possess some metric or other, the particular class of metrics (1) holding if it is in fact the case that the aggregate of nebulæ satisfies the homogeneity conditions set out above. If it should turn out that

* Purely as a matter of mathematical convenience, $t$ and $r$ are both measured in the astronomical unit of distance, the parsec, which is $3.08 \times 10^{18} \mathrm{~cm}$. The time in years is $t / c$, where $c$ is the velocity of light expressed in parsecs per year. 